\title{
ORIGINAL ARTICLE \\ Genetic interactions controlling sex and color establish the potential for sexual conflict in Lake Malawi cichlid fishes
}

\author{
NF Parnell and JT Streelman \\ Sex-determining systems may evolve rapidly and contribute to lineage diversification. In fact, recent work has suggested \\ an integral role of sex chromosome evolution in models of speciation. We use quantitative trait loci analysis of restriction \\ site-associated DNA -tag single nucleotide polymorphisms to identify multiple loci responsible for sex determination and \\ reproductively adaptive color phenotypes in Lake Malawi cichlids. We detect a complex epistatic sex system consisting of a \\ major female heterogametic ZW locus on chromosome 5, two separate male heterogametic XY loci on chromosome 7, and two \\ additional interacting loci on chromosomes 3 and 20. Our data support the known chromosomal linkage between orange blotch \\ color and ZW, as well as novel genetic associations between male blue nuptial color and two sex determining regions (an XY \\ and ZW locus). These results provide further empirical evidence for a complex antagonistic sex-color system in this species \\ flock and suggest a possible role for, and effect of, polygenic sex-determining systems in rapid evolutionary diversification. \\ Heredity (2013) 110, 239-246; doi:10.1038/hdy.2012.73; published online 24 October 2012
}

Keywords: sex determination; speciation; sexual antagonism; RAD-tag; QTL; cichlid

\section{INTRODUCTION}

One of the most fundamental and surprisingly diverse processes in the life history of an organism is the determination of sex. An array of environmental and genetic controls has been discovered across divergent taxa, yet in the majority of cases the sex-determining loci remain unidentified. Although sex-determining systems are frequently associated with a sex-specific heteromorphic chromosome pair (that is, male $\mathrm{XY}$ or female $\mathrm{ZW}$ ), more cryptic and complex systems are widespread (Ezaz et al., 2006). For example, among teleost fishes one finds both simple and polygenic sex determination, yet no general patterns exist and even the master sex-determining loci may vary within genera (Matsuda et al., 2002; Nanda et al., 2002; Mank et al., 2006; Yoshida et al., 2011). The maintenance of functional diversity in such an integral genetic and developmental system sets sex determination apart from other more highly conserved pathways (Wilkins, 1995; Carroll, 2000).

The importance of reproductive isolation in speciation has led to interest in the role of intersexual conflict, and sex-determining systems, in this process (Kitano et al., 2009; Qvarnstrom and Bailey, 2009; Meiklejohn and Tao, 2010). Sexually antagonistic selection is known to produce intersexual conflict (Rice, 1992, 1998; Chapman, 2006) and possible resolution of such conflict involves the evolution of sex-determining loci linked to alleles favoring male- or femalespecific traits (Kocher, 2004; Streelman et al., 2007). Lande et al. (2001) incorporated color-based sexual selection and sexual conflict into speciation models, with cichlid fishes in mind. In this conception, a dominant female sex determiner (W) invades an XY male heterogametic system, reinforced by a W-linked novel color and unlinked loci for mate preference. Divergence is achieved in sympatry by assortative mating through a complex interplay of new sex systems, repressors/modifiers of sex determination and linked color patterns.
Kocher (2004) envisions a complementary scenario in which new sexdetermining systems and associated sex-specific color patterns are favored during different stages of population growth, after founder events due to changes in lake levels. Together, these models suggest (i) the evolution of new sex-determining systems linked to male- or female-specific traits (that is, color patterns) and (ii) the possibility of a 'speciation engine' (Kocher, 2004) as assortative mating by novel color patterns resolves sexual antagonism. In theory, it is possible that new and old sex-determining loci might remain polymorphic within and between populations, providing standing variation for interacting sex-determining systems (Wilkins, 1995; van Doorn and Kirkpatrick, $2007,2010)$. The evolutionary dynamics of sex systems as a result of sexually antagonistic selection has been suggested as important for the high speciation rates of some fishes (Seehausen et al., 1999a; Lande et al., 2001; Kocher, 2004; Ross et al., 2009), including the cichlids of Lake Malawi (LM), East Africa.

The LM cichlid flock consists of hundreds of species that have arisen in the past 500000 to 1000000 years, presumably the fastest and the most extensive vertebrate adaptive radiation known (Won et al., 2005; Seehausen, 2006). The rapidity of this radiation, extreme endemism (Parnell and Streelman, 2011) and continued low levels of gene flow between species (Mims et al., 2010) has led to a phenomenon wherein genetic incompatibilities between lineages are considerably outpaced by speciation (Stelkens et al., 2010). The integrity of species is maintained by pre-zygotic mechanismsassortative mating based on visual (Seehausen et al., 1997, 2008; van Oppen et al., 1998; Knight and Turner, 2004; Maan et al., 2004; Carleton, 2009), auditory (Amorim et al., 2004, 2008; Simoes et al., 2008; Smith and van Staaden, 2009; Danley et al., 2011) and olfactory cues (Jordan et al., 2003; Plenderleith et al., 2005; Cole and Stacey, 2006) that sometimes breakdown. Interspecific and intergeneric 
hybridization has been well documented within all of the African cichlid species flocks, including LM (Ruber et al., 2001; Salzburger et al., 2002; Smith and Kornfield, 2002; Smith et al., 2003; Streelman et al., 2004; Gerlai, 2007; Mims et al., 2010). Hybridization among such young species often produces viable, fertile offspring and maintains standing genetic and phenotypic variation (that is, the 'hybrid swarm' hypothesis, Seehausen, 2004; Albertson and Kocher, 2005; Genner and Turner, 2005; Loh et al., 2008; Parnell et al., 2008). In fact, researchers exploit this situation, where species behave genetically like populations, by designing interspecific (or intergeneric) crosses to identify links between genotype and phenotype (Streelman et al., 2003, Albertson et al., 2005; Parnell et al., 2012).

Using this strategy, Streelman, Albertson and Kocher mapped the genetic basis of orange-blotch $(\mathrm{OB})$ color to linkage group 5 in an intergeneric cross between Labeotropheus fuelleborni and Metriaclima zebra, two members of LM's rock-dwelling lineage of species (Streelman et al., 2003). They showed that OB was presumably linked to a female heterogametic sex determiner (W), while a male heterogametic locus (Y) was observed on a different chromosome (Albertson, 2002). The segregation of (i) OB on linkage group 5 within multiple species of rock-dwelling Malawi cichlids (Roberts et al., 2009), as well as (ii) both sex-determining systems within species of the genus Metriaclima (Ser et al., 2010) has been confirmed using selective screening of color- and/or sex-associated markers. The study by Ser et al. (2010) is particularly notable here because it demonstrated that some Metriaclima species segregate both XY and ZW sex systems and also speculated that other sex determiners must be present in the genomes of LM rock-dwelling cichlids.

The goal of our study is twofold. First, we aim to identify additional loci contributing to sex determination and color pattern in LM rock-dwelling cichlids and to ask whether these, as well as previously identified loci, segregate in unstudied rock-dwelling species. Second, we develop an experimental design powered to quantify, at a genome-wide scale, the genetic contributions and interactions among loci with effects on sex and color. Using quantitative trait loci (QTL) analysis of restriction site-associated DNA (RAD)-tag single nucleotide polymorphisms (SNPs) in a new $\mathrm{F}_{2}$ hybrid cross, we identified five genomic regions (and epistatic interactions) contributing to sex determination in LM rock-dwelling cichlids. We confirm the association between a major ZW locus and $\mathrm{OB}$ color and highlight novel linkage between male nuptial color and both the ZW plus a male-heterogametic XY system. Our results are bolstered by consistency with previous work in different LM rockdwelling species and contribute to an overall understanding of the interplay between sex and color genetic systems in this young species assemblage.

\section{MATERIALS AND METHODS}

\section{Production of $F_{2}$ to map sex and color in new species}

We chose two LM cichlid species from the rock-dwelling (mbuna) clade, Cynotilapia afra and Pseudotropheus elongatus, in which sex determination has not been studied previously. These species co-occur at several sites across the lake and differ in a number of phenotypes of interest, including craniofacial morphology, coloration, tooth shape and density and gut length. Fishes collected from LM were acquired from Old World Exotics fish suppliers (Miami, FL) and several C. afra males were placed in a 189-L aquarium with P. elongatus females at a ratio of 1:2. Within a few weeks, the dominant male (and subsequent sire) had established an obvious hierarchy over his conspecifics and had fertilized the eggs of the dam. Fin clips $\left(25 \mathrm{~mm}^{2}\right)$ were taken from both the dam and sire for RAD-tag library preparation and the pair was kept in a breeding tank with dither fish to diffuse male aggression. Offspring were taken from the dam after hatching, grown up in net pens and moved to consecutively larger aquaria as they grew. This pair produced several $\mathrm{F}_{1}$ broods, which were maintained and intercrossed $\left(\mathrm{F}_{1} \times \mathrm{F}_{1}\right)$ in 189-L aquaria for 2 years during which time they produced over 40 broods totaling some $600 \mathrm{~F}_{2}$ offspring (mean brood size $=15$ ). Each $\mathrm{F}_{2}$ family was isolated and numbered by brood sequence. A total of $397 \mathrm{~F}_{2}$ hybrids were euthanized (tricaine methane sulfonate, MS-222, in accordance with established Georgia Institute of Technology IACUC protocols) and processed for DNA samples and a suite of morphological traits (for example, below; Parnell et al., 2012). When sampled, individuals were at least $75 \mathrm{~mm}$ total length (approximately 1-year old).

\section{Color assignment and sex determination}

Following euthanasia, hybrid individuals were photographed in a full-body lateral view for color assignment. The C. afra sire (Mara yellow type) had yellow body color with black bars (for example, Figure 1a). The P. elongatus dam exhibited the yellow-orange ' $O$ ' color, putatively the homozygous morph of OB that lacks black blotches (Figure 1i; Lande et al., 2001). All $\mathrm{F}_{1}$ hybrid individuals were $\mathrm{OB}$. We used binary scoring to map the genetic basis of $\mathrm{OB}$ (Supplementary Figure $\mathrm{S} 1$ ); $\mathrm{F}_{2}$ individuals were assigned to two color classes, 'OB' where 'O' is also scored OB (1) or 'not OB' (0). We observed a blue color among male $F_{2}$ that was not seen in either of the parental individuals (see Figure 1) but is a known nuptial phenotype common in these and other LM species (Ribbink et al., 1983; Svensson et al., 2011). To map the genetic basis of blue color, we assigned $F_{2}$ individuals to two categories, 'blue' (1) or 'not blue' (0). Note that with this assignment scheme, it is possible for fish to be both OB and blue (Figure 1f; Supplementary Figure S1) and in fact this phenotype is observed in males of $P$. elongatus. Fish were dissected and sex was determined from gross morphology (all fish were sexually mature) and assigned binary coding as male (1) or female (0).

DNA extraction, RAD library preparation and Illumina Sequencing Malawi cichlids have highly similar genomes and share polymorphism across population, species and major lineage boundaries; for instance, the nucleotide diversity observed across survey-sequenced genomes from five diverse representatives of the species flock was less than that among laboratory strains of zebrafish (Loh et al., 2008). Therefore, we sought a strategy to identify informative markers for mapping before the genotyping phase of our study. Genomic DNA was purified from sire and dam fin tissue using the DNeasy Blood and Tissue Kit (Qiagen, Valencia, CA, USA) and RAD-tag libraries were prepared and sequenced from each as described elsewhere (Parnell et al., 2012). The RAD libraries for each parent were assembled, aligned and alternately fixed SNPs were identified and selected for genotyping in the intercross population $\left(\mathrm{F}_{2}\right)$.

\section{SNP genotyping in $\mathrm{F}_{2}$ individuals}

A total of $382 \mathrm{~F}_{2}$ individuals as well as sire and dam were genotyped at 384 SNP markers by the Emory Biomarker Service Center (Emory University). The Illumina (San Diego, CA, USA) BeadArray genotyping platform was coupled with the GoldenGate assay (Illumina) in which oligonucleotide pool assays are designed specifically to discriminate between alleles at a SNP (Oliphant et al., 2002; Fan et al., 2003). Marker genotypes were examined to evaluate accuracy of initial sequencing during SNP discovery and 12 loci were removed because they did not segregate between the parents. Another 12 markers that contained one heterozygous parental genotype were removed, as well as seven others with low genotyping success in either parental or $\mathrm{F}_{2}$ individuals. This left a fully informative set of 353 SNP markers, with nearly complete genotypic data across the $\mathrm{F}_{2}$ population ( $0.6 \%$ missing data).

\section{Genetic linkage map construction}

A genetic linkage map was produced with SNP marker genotype data using JoinMap 3.0 (Dyazma, Wageningen Netherlands) software (van Ooijen and Voorrips, 2001), as described (Parnell et al., 2012). The map was created using Kosambi's mapping function, a logarithm of the odds (LOD) threshold of 1.0, a recombination threshold of 0.4 , a jump threshold of 5.0 and a ripple function with no fixed order of loci. A LOD threshold of 4.0 was used to join 344 loci in 22 linkage groups with a total map size of $1082 \mathrm{cM}$ and average marker distance $3.67 \pm 1.71 \mathrm{cM}$. To facilitate comparison to other genetic maps in Malawi (Streelman et al., 2003; Albertson et al., 2005) as well as tilapiine 

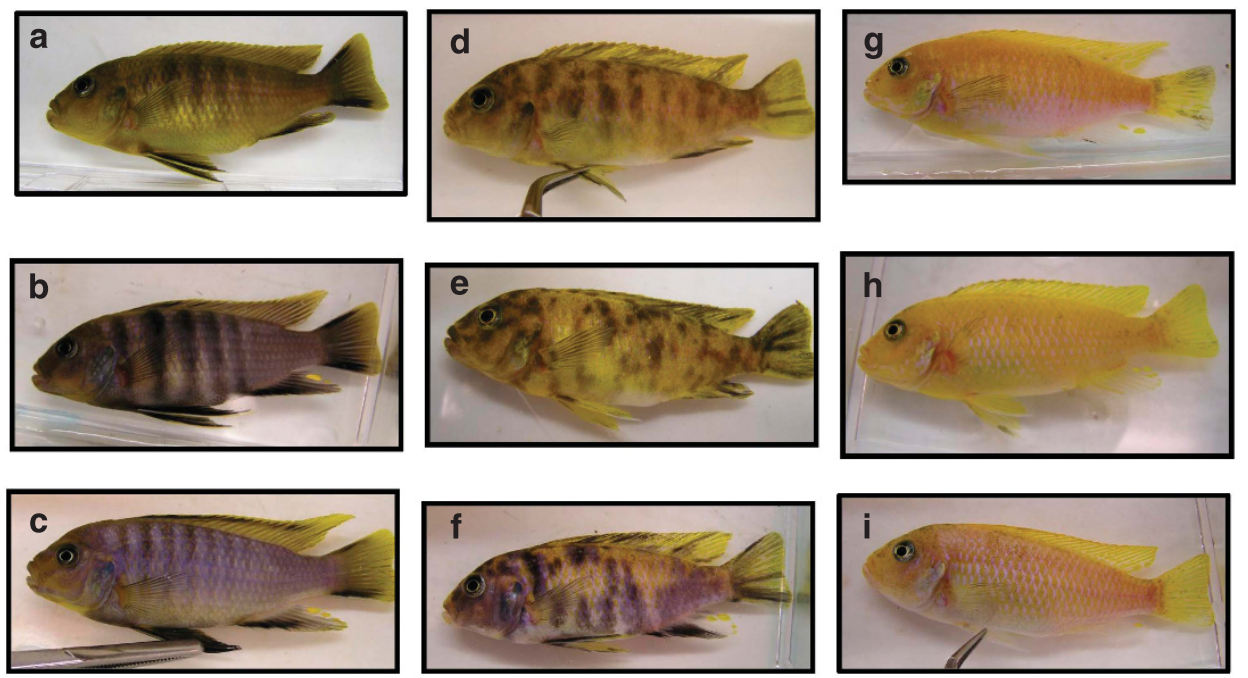

Figure 1 Representative color phenotypes of $F_{2}$ hybrid individuals. (a) Representation of the sire color pattern, Cynotilapia afra (although this is a $F_{2}$ individual pictured) and (i) displays the dam, Pseudotropheus elongatus ('O' phenotype=homozygous WW) phenotype. (b), (c) and (f) show individuals scored as 'blue' with (b) and (c) being blue-barred and (f) as blue-blotched ('blue-OB'). Individuals (d), (e) and (g-i) represent the 'OB' phenotype with (g-i) displaying the ' $O$ ' color (homozygous WW), which does not occur in males. (d) and (e) are OB individuals representative of the phenotype of all $F_{1}$ individuals produced.

cichlids (Lee et al., 2005), our linkage group names represent consensus cichlid chromosomes.

\section{QTL mapping of sex and color}

The linkage map was used to determine genomic locations for sex, OB and blue color in the $\mathrm{F}_{2}$ population using the R/qtl package (Broman and Sen, 2009). We used an iterative approach by scanning for single QTL with standard and composite interval mapping, followed by two-dimensional scans to (i) identify QTL by QTL interactions and (ii) detect additional QTL. Finally, using results of the previous steps we built multiple QTL models incorporating QTL interactions and covariates. In the multiple QTL model process, we used a forward-backward selection algorithm to add and remove QTL based on overall model effects and the effects of single QTL as they were removed from the model. Genotype-phenotype associations are scored using the LOD, which represents the $\log _{10}$ likelihood ratio comparing the hypothesis of a QTL at a marker location to the null hypothesis of no QTL $\left(\mathrm{LOD}=(\mathrm{n} / 2) \log _{10}\left(\mathrm{RSS}_{0} /\right.\right.$ $\mathrm{RSS}_{1}$ ); RSS = residual sum of squares, Broman and Sen, 2009). The variance in a phenotype is assigned to each significant QTL (or covariate) and reported as percentage variance explained (PVE) in the analysis output. The total variance accounted for by QTL is a proxy for the heritability of a trait and is calculated as $1-10^{-(2 / n) L O D}$ (Broman and Sen, 2009). Significance thresholds for LOD scores were estimated using 1000 permutations of phenotypes relative to genotypes to build a distribution of maximum genome-wide LOD scores. From this distribution, the 95th percentile LOD score was calculated to serve as a threshold for significant QTL associations (Broman and Sen, 2009). Due to the discrete nature of our data set, we used the Haley-Knott regression mapping method and binary phenotype modeling.

\section{RESULTS}

Sex

Our dataset for the analysis of sex included 353 individuals, with complete phenotype data exhibiting a sex ratio of 58:42 female to male. Two QTL of major effect were detected on chromosomes 5 and 7 during the single-locus scan for sex (Figure 2). Simultaneously scanning for two QTL plus interactions supported these loci and indicated a second locus on chromosome 7 exhibiting epistasis, with the major effect locus on chromosome 5. Using the results of the single and double scans, a multiple QTL model was constructed and

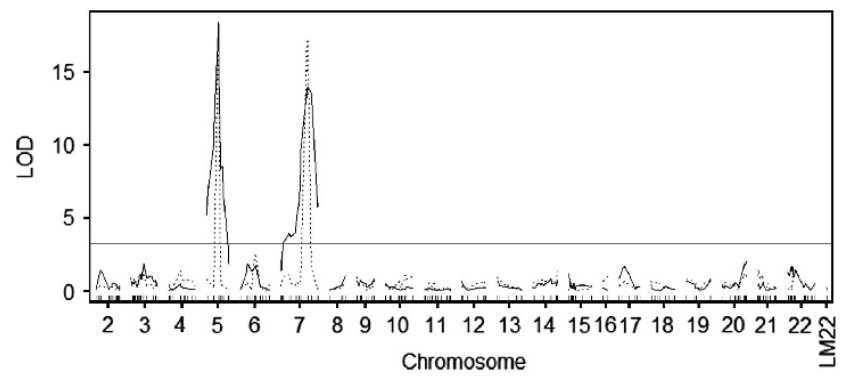

Figure 2 Single QTL scans for loci affecting sex. The solid line indicates standard interval mapping and dashed line indicates composite interval mapping. The horizontal axis denotes chromosome number with hash marks representing marker positions; vertical axis is log-likelihood score (LOD) of marker association with phenotype. The horizontal bar indicates significant LOD score (3.5; $P<0.05)$.

tested in multiple iterations, producing a significant final model $(\mathrm{LOD}=53.36)$. The full model incorporated five loci, three epistatic effects and accounted for over $50 \%$ of variance in sex $(\mathrm{PVE}=51.5$; see Table 1).

In the multiple QTL model, the largest effect was seen at position $26.3 \mathrm{cM}$ on chromosome 5 (5@26.3), explaining an estimated 23.5\% of the phenotypic variance and matching the precise position indicated in single and double QTL scans. This region of chromosome 5 has previously been suggested as the location of a ZW sexdetermining system in which the female allele (W) is dominant (Streelman et al., 2003; Ser et al., 2010). The QTL behaves in a roughly similar fashion here (Figure 3 and Table 2). Individuals with WW genotypes were always female, regardless of genotypes at other loci, while sex ratios (fraction of females) for ZW and ZZ genotypes were 0.54 and 0.43 , respectively. The second large-effect locus (7@67.2) accounted for $10.83 \%$ of the variance $(\mathrm{LOD}=14.88)$ in sex (Table 1), and its action and position suggests that it represents an XY system (Ser et al., 2010). The major ZW locus is semi-dominant 
in sex determination to this $\mathrm{XY}$ and all other loci; two $\mathrm{W}$ alleles at the ZW locus trump any combination of alleles elsewhere in the genome. However, the sex of ZW and ZZ individuals depends strongly upon genotypes at 7@67.2, as well as those at other loci (Figure 3 and below).

An unlinked QTL identified on chromosome 20 (20@28.1) exhibited significant effects in the model $(\mathrm{LOD}=11.44, \mathrm{PVE}=8.14)$

Table 1 Results of multiple QTL mapping (MQM) for factors controlling sex

\begin{tabular}{lcccc}
\hline & $d f$ & LOD & PVE & P-value $\left(\chi^{2}\right)$ \\
\hline Full model & 25 & 53.36 & 51.46 & $<0.000001$
\end{tabular}

Drop one QTL at a time ANOVA table:

\begin{tabular}{|c|c|c|c|c|}
\hline & $d f$ & $\angle O D$ & PVE & P-value $\left(\chi^{2}\right)$ \\
\hline $5 @ 26.3(Z W)$ & 6 & 29.15 & 23.50 & $<0.000001$ \\
\hline 7@67.2 (XY) & 6 & 14.88 & 10.83 & $<0.000001$ \\
\hline $20 @ 28.1\left(X^{\prime} Y^{\prime}\right)$ & 12 & 11.44 & 8.14 & $<0.000001$ \\
\hline 3@27.0 ( $\left.Z^{\prime} W^{\prime}\right)$ & 6 & 6.30 & 4.32 & 0.000006 \\
\hline $7 @ 44.2\left(X^{\prime \prime} Y^{\prime \prime}\right)$ & 6 & 5.80 & 3.97 & 0.000164 \\
\hline brood & 3 & 3.75 & 2.53 & 0.0006 \\
\hline 20@28.1:7@67.2 & 4 & 6.10 & 4.18 & 0.000012 \\
\hline 3@27.0:20@28.1 & 4 & 5.24 & 3.57 & 0.000075 \\
\hline 5@26.3:7@44.2 & 4 & 4.09 & 2.77 & 0.000842 \\
\hline 20@28.1:brood & 2 & 1.86 & 1.24 & 0.013884 \\
\hline
\end{tabular}

Abbreviations: ANOVA, analysis of variance; LOD, logarithm of the odds; PVE, percentage variance explained; QTL, quantitative trait loci.

The log-likelihood score (LOD), PVE, and the $X^{2} P$-value for the full model are indicated in the top portion. The lower portion of the table represents the results of dropping a factor from the model one at a time. Putative QTL are indicated as chromosome@position in centimorgans (cM), 'brood' represents inclusion of family as a cofactor and epistatic interactions are indicated by factor:factor notation. Significant results are indicated as ${ }^{*} P<0.05,{ }^{*} P<0.005$ and $* * * P<0.0005$ and epistasis with XY (Table 1). This locus behaves similarly to the putative $\mathrm{XY}$ (thus is labeled $\mathrm{X}^{\prime} \mathrm{Y}^{\prime}$ ) and interacts with a fourth QTL on chromosome3 (3@27.0) that subsequently behaves like a second ZW (thus labeled $\mathrm{Z}^{\prime} \mathrm{W}^{\prime}$ ). A fifth QTL (7@44.2) was detected $23 \mathrm{cM}$ proximal to the putative XY (7@67.2). Genotypes here are similar to those at the distal XY site due to linkage; however, they differ in $37 \%$ of $\mathrm{F}_{2}$ individuals. The behavior of this locus in sex determination appears to be as a complementary effector to the $\mathrm{XY}$, functioning as another male determiner (thus labeled $X^{\prime \prime} Y^{\prime \prime}$ ). Epistatic interactions had modest variance effects and relatively low LOD scores, yet they are integral to the strength of the overall model as well as the explanation of the effects of haplotype on sex (Figure 3). A significant effect of $F_{2}$ family ('brood') was detected in this analysis. This is most likely due to skewed sex ratios in a handful of families (4 skewed female, 1 skewed male) as well as a single family that was entirely male. Parentage (individual identity of mother or father) of $F_{2}$ fish had no effect on sex when held as a covariate.

\section{Color}

Color phenotypes were available for $340 \mathrm{~F}_{2}$ individuals. We mapped two color traits, orange blotch $(\mathrm{OB})$ and blue (Figure 1 and

Table 2 Association between sex and genotype at the putative ZW locus (chromosome 5 at $26.3 \mathrm{cM}$ )

\begin{tabular}{lccc}
\hline & $Z Z$ & $Z W$ & $W W$ \\
\hline Male & $57 \%$ & $46 \%$ & 0 \\
Female & $(n=52)$ & $(n=82)$ & \\
& $43 \%$ & $54 \%$ & $100 \%$ \\
& $(n=40)$ & $(n=95)$ & $(n=69)$ \\
\hline
\end{tabular}

All homozygous ' $W$ ' individuals are female, while heterozygotes were slightly skewed towards females and homozygous ' $Z$ ' individuals were slightly skewed towards males, due to addition loci and their interactions.

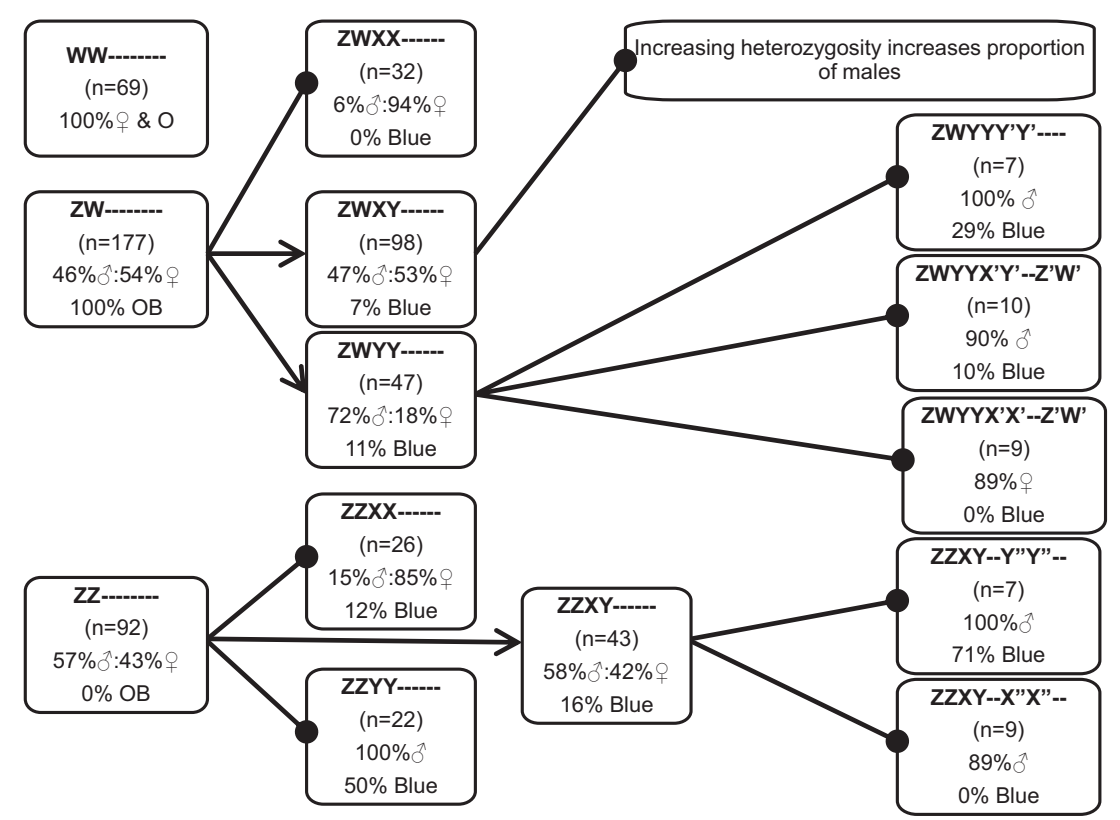

Figure 3 Patterns in sex and color phenotypes based on associated haplotypes at loci identified in multiple QTL models (see text). Locus order in the figure is as follows: $Z W \rightarrow X Y \rightarrow X^{\prime} Y^{\prime} \rightarrow X^{\prime \prime} Y^{\prime \prime} \rightarrow Z^{\prime} W^{\prime}$ (see Table 1). Locus positions are: $Z W=5 @ 26.3, \quad X Y=7 @ 67.2, \quad X^{\prime} Y^{\prime}=20 @ 28.1, \quad X^{\prime \prime} Y^{\prime \prime}=7 @ 44.2$, $Z^{\prime} W^{\prime}=3 @ 27.0$. Arrowheads indicate that haplotype patterns are further resolved at the next step while filled circles indicate the final level of resolution. Dashes within a haplotype represent locus genotypes that do not show a clear effect on the phenotype. 
Table 3 Results of multiple QTL mapping (MQM) for factors controlling the orange blotch $(\mathrm{OB})$ color pattern

\begin{tabular}{lcccc}
\hline & $d f$ & $L O D$ & PVE & P-value $\left(\chi^{2}\right)$ \\
\hline Full model & 5 & 311.82 & 98.54 & $<0.000001$
\end{tabular}

Drop one QTL at a time ANOVA table:

\begin{tabular}{lrrrcr}
\hline & $d f$ & $L O D$ & PVE & P-value $\left(\chi^{2}\right)$ & Sig. \\
\hline $5 @ 26.3$ & 4 & 308.58 & 94.24 & $<0.000001$ & $* * *$ \\
sex & 3 & 0.36 & 0.01 & 0.655 & \\
$5 @ 26.3$ sex & 2 & 0.12 & 0.00 & 0.761 & \\
\hline
\end{tabular}

Abbreviations: ANOVA, analysis of variance; LOD, logarithm of the odds; PVE, percentage variance explained; QTL, quantitative trait loci.

The log-likelihood score (LOD), PVE and the $\chi^{2} P$-value for the full model are indicated in the top portion. The lower portion of the table represents the results of dropping a factor from the model one at a time. Putative QTL are indicated as chromosome@position in centimorgans (cM), 'sex' represents inclusion of sex as a cofactor and epistatic interactions are indicated by factor:factor notation. Significant results are indicated as ${ }^{*} P<0.05,{ }^{* *} P<0.005$ and $* * * P<0.0005$

Supplementary Figure S1), in separate analyses. Approximately $75 \%$ of $\mathrm{F}_{2}$ individuals were $\mathrm{OB}$ ( $\mathrm{or}$ ' $\mathrm{O}$ '). Notably, $100 \%$ of $\mathrm{F}_{1}$ individuals exhibited the $\mathrm{OB}$ phenotype. Previous work has indicated a single large-effect QTL for the OB phenotype on cichlid chromosome 5, explained by alleles at the pax7 locus (Streelman et al., 2003; Roberts et al., 2009). This QTL was detected in each step of our analysis and found to account for over $90 \%$ of the phenotypic variance in $\mathrm{OB}$ color pattern (Table 3, Supplementary Figure S2). OB (5@26.3) is mapped coincident with the inferred $\mathrm{ZW}$ sex-determining locus (above). The presence of a single $\mathrm{W}$ allele is sufficient to express $\mathrm{OB}$ in all individuals, while homozygous WW individuals exhibit the 'O' phenotype.

The blue phenotype was an emergent trait in this cross between a homozygous OB ('O') dam and a black-barred yellow sire (see Figure 1). 'Blue' individuals included those with blue/orange blotches (approximating a blue OB pattern; a male phenotype in P. elongatus) as well as fish with black bars on blue background (similar to 'BB' or 'blue-barred' in Streelman et al. (2003); see Supplementary Figure S1). Ten percent of $\mathrm{F}_{2}$ exhibited blue color with the majority being ' $\mathrm{BB}$ ' type. Our analysis revealed several factors associated with this phenotype (full model LOD $=63.25$; Table 4, Supplementary Figure S3). The largest factor contributing to blue was the covariate sex (only males are blue; PVE $=19.77$ ) while the largest PVE (8.70) for a putative QTL was at position 44.2 on chromosome 7 (Table 4). There were no effects of $F_{2}$ family ('brood') or parentage on this trait. We detected a handful of loci of moderate effect on several chromosomes, as well as epistatic interactions between QTL and sex (Table 4). The QTL for blue color on chromosome 7 (position 44.2) is coincident with an XY sex determiner ( $\mathrm{X}^{\prime \prime} \mathrm{Y}^{\prime \prime}$ above). A second locus of particular interest for the blue phenotype is found on chromosome $5,1.6 \mathrm{cM}$ away from $\mathrm{OB} / \mathrm{ZW}$. Just as the $\mathrm{OB}$ polymorphism has been linked to sex-determining loci in Malawi cichlids (Streelman et al., 2003; Roberts et al., 2009; Ser et al., 2010), here we observe a similar phenomenon for blue color.

\section{DISCUSSION}

Diverse sex systems in diverse cichlids

Sex-determining loci are important in evolution because they may (i) resolve sexual conflict between traits favored by selection in males versus females (for example, color patterns), (ii) evolve rapidly and
Table 4 Results of multiple QTL mapping (MQM) for factors controlling blue color

\begin{tabular}{lcccc}
\hline & $d f$ & LOD & PVE & P-value $\left(\chi^{2}\right)$ \\
\hline Full model & 31 & 63.25 & 57.54 & $<0.000001$
\end{tabular}

Drop one QTL at a time ANOVA table:

\begin{tabular}{lrrrrr}
\hline & $d f$ & $L O D$ & PVE & P-value $\left.\chi^{2}\right)$ & Sig. \\
\hline sex & 9 & 28.22 & 19.77 & $<0.000001$ & $* * *$ \\
7@44.2 & 10 & 13.76 & 8.70 & $<0.000001$ & $* * *$ \\
LM22@0.8 & 4 & 13.53 & 8.54 & $<0.000001$ & $* * *$ \\
5@27.9 & 6 & 10.08 & 6.21 & $<0.000001$ & $* * *$ \\
14@42.1 & 4 & 9.85 & 6.06 & $<0.000001$ & $* * *$ \\
6@43.8 & 4 & 8.79 & 5.37 & $<0.000001$ & $* * *$ \\
15@15.7 & 4 & 7.29 & 4.41 & $<0.000001$ & $* * *$ \\
18@39.8 & 6 & 5.77 & 3.45 & $<0.000001$ & $* * *$ \\
sex:LM22@0.8 & 2 & 8.41 & 5.13 & $<0.000001$ & $* * *$ \\
5@27.9:7@44.2 & 4 & 8.05 & 4.89 & $<0.000001$ & $* * *$ \\
sex:14@42.1 & 2 & 6.38 & 3.83 & $<0.000001$ & $* * *$ \\
sex:6@43.8 & 2 & 5.78 & 3.46 & $<0.000001$ & $* * *$ \\
7@44.2:18@39.8 & 4 & 4.11 & 2.43 & 0.001 & $* *$ \\
sex:15@15.7 & 2 & 4.07 & 2.40 & $<0.000001$ & $* * *$ \\
\hline
\end{tabular}

Abbreviations: ANOVA, analysis of variance; LOD, logarithm of the odds; PVE, percentage variance explained; QTL, quantitative trait loci.

The log-likelihood score (LOD), PVE and the $X^{2} P$-value for the full model are indicated in the top portion. The lower portion of the table represents the results of dropping a factor from the model one at a time. Putative QTL are indicated as chromosome@position in centimorgans (cM), 'sex' represents inclusion of sex as a cofactor and epistatic interactions are indicated by factor:factor notation. Significant results are indicated as ${ }^{*} P<0.05,{ }^{* *} P<0.005$ and factor:factor not

contribute to reproductive isolation and/or (iii) act as genomic attractors for genes controlling phenotypes subject to natural selection. This is particularly true in lineages where multiple, recently evolved sex determiners segregate as polymorphism, and sex chromosomes are in the early stages of evolution (Streelman et al., 2007). LM rock-dwelling cichlids are ideal for investigating sex determination due to the existence of multiple sex systems not only between but also within species (Albertson, 2002; Streelman et al., 2003; Ser et al., 2010). The Malawi radiation has been so rapid that biological species continue to exchange genes by hybridization (for example, Mims et al. (2010)) and major intrinsic post-zygotic incompatibilities separate only the most divergent lineages (Stelkens et al., 2010).

Using QTL analysis of RAD-tag SNPs, we have identified and characterized the (additive and epistatic) effects of five sex-determining regions in a single hybrid cross between LM rock-dwelling cichlid species. We mapped the genetic basis of two color phenotypes with putatively conflicting roles in male versus female fitness (see below; Holzberg, 1978; Ribbink et al., 1983; van Oppen et al., 1998; Konings, 2007; Roberts et al., 2009; Svensson et al., 2011) and established the chromosomal linkage of each to XY and/or ZW sex loci. Our results support the concept that cichlid chromosome 5 hosts a female heterogametic (ZW) locus coincident with QTL for (female-associated) OB patterning (Streelman et al., 2003; Roberts et al., 2009; Ser et al., 2010), as well as male-associated blue color. We find strong evidence for additional sex-determining loci, including two male heterogametic ( $\mathrm{XY} \& \mathrm{X}^{\prime \prime} \mathrm{Y}^{\prime \prime}$, where $\mathrm{X}^{\prime \prime} \mathrm{Y}^{\prime \prime}$ is linked to blue color) loci on chromosome 7 , plus complimentary $\mathrm{X}^{\prime} \mathrm{Y}^{\prime}$ and $\mathrm{Z}^{\prime} \mathrm{W}^{\prime}$ loci on unlinked chromosomes (20 and 3, respectively). Taken together, these QTL account for over $50 \%$ of the variance in sex in this pedigree $\left(\mathrm{LOD}=53.36, P<10^{-10}\right)$. 
Our data are informative to consider in the context of a recent study by Ser et al. (2010). In that analysis, families of multiple rockdwelling Malawi cichlids from the genus Metriaclima were screened for the effects of putative sex determiners on chromosomes 5 and 7 (Albertson, 2002; Streelman et al., 2003). These sex systems were shown to be widespread among Metriaclima populations and to interact epistatically in some families. Importantly, these loci alone could not account for sex in all families, leading to the prediction that additional sex determiners segregate across the Malawi flock. Our analysis addresses that prediction and pinpoints three additional putative sex determiners in Malawi rock-dwelling cichlids. Our quantitative genetic approach similarly allows the partitioning of phenotypic variance to each locus and formally evaluates the epistatic relationships between the segregating sex systems. Notably, the power of our intercross design to accurately model phenotypic effects (including moderate-effect loci) and epistasis is the product of numerous fully informative SNP loci and nearly complete genotypic plus phenotypic data for $\sim 350 \mathrm{~F}_{2}$ (Broman and Sen, 2009).

\section{A complex polygenic system for sex}

We used the inferred mode of action, epistatic interactions and phenotypic variance explained by sex QTL to arrange these loci in a hierarchical scheme of sex determination (Figure 3). All WW (5@26.3) individuals were female (20.4\% of individuals), while ZW and $\mathrm{ZZ}$ genotypes showed slightly skewed sex ratios (female:male 54:46\%:43:57\%, respectively). The expectation in a purely ZW system is that the $\mathrm{W}$ allele confers female sex, yet we do not observe this pattern; only homozygous $\mathrm{W}$ genotypes are unanimously female. This discrepancy highlights the importance of additional loci affecting sex and the genetic interactions contributing to sex determination in Malawi cichlids.

The sex of ZW individuals is strongly influenced by genotype at the distal chromosome 7 QTL (7@67.2), which we call the primary XY ZWXX individuals are predominantly female (94\%), ZWYY are mostly male $(72 \%)$ and ZWXY individuals showed a roughly equal sex ratio (53:47\% female to male). Notably, all but 3 of the $82 \mathrm{ZW}$ males are XY or YY at a minimum of two of the three putative XY loci $\left(X Y, X^{\prime} Y^{\prime}, X^{\prime \prime} Y^{\prime \prime}\right)$, and over half are $X Y$ or YY at all three. Similarly, $X Y$ genotypes influence the sex of ZZ fishes; ZZYY individuals are all male, while ZZXX $F_{2}$ are $85 \%$ female. Additional QTL for sex have a refining role in sex determination and/or act to 'settle ties' between the primary ZW and XY loci. For instance, ZWYY individuals who inherit a $Y^{\prime} Y^{\prime}$ genotype from the putative $X^{\prime} Y^{\prime}$ locus (20@28.1) are $100 \%$ male. ZWYY individuals who inherit an $X^{\prime} Y^{\prime}$ from 20@28.1 plus a $\mathrm{Z}^{\prime} \mathrm{W}^{\prime}$ from the putative second ZW locus at 3@27 are predominantly male (90\%); however, ZWYYX' $\mathrm{X}^{\prime}-\mathrm{Z}^{\prime} \mathrm{W}^{\prime}$ genotypes are $89 \%$ female. Likewise, the sex of ZZXY individuals is influenced by genotypes at the $\mathrm{X}^{\prime \prime} \mathrm{Y}^{\prime \prime}$ locus (7@44.2) such that $\mathrm{ZZXY}-\mathrm{Y}^{\prime \prime} \mathrm{Y}^{\prime \prime}$ fishes are $100 \%$ male.

Because our $\mathrm{F}_{2}$ population is finite in size, certain genotypic combinations did not appear frequently enough to quantitatively evaluate the effects and/or interactions among component loci. However, modeling sex determination in the context of QTL haplotypes (Figure 3 ) allows us to explain sex in $\sim 82 \%$ of $\mathrm{F}_{2}$ offspring. We cannot discount the effect of environmental influences on sex determination in fishes (Baroiller et al., 2009), and we accept that these effects in cichlids in particular are not well described. The conditions in which we conducted our intercross experiment were well controlled in terms of light, temperature and other physical water parameters. However, social, dietary and yet unmeasured factors may still account for variance in sex, such as the small but significant effect of brood in our analysis (Table 1).

\section{Sex, color and their co-evolution}

Our analysis provides evidence for sex linkage of colors and patterns expected to favor male (blue color) versus female (OB pattern) fitness. The coincidence of QTL for OB color with the female-dominant ZW has been suggested by previous work (Streelman et al., 2003; Roberts et al., 2009; Ser et al., 2010). Here, the W allele confers this color phenotype to all individuals possessing it. Of $246 \mathrm{OB}$ (and O) individuals, 164 were female $(66.7 \%)$ and all $(100 \%)$ were genetically ZW (or WW), while the $82 \mathrm{OB}$ males were exclusively ZW. OB has been suggested to function in disruptive crypsis, thereby conferring an advantage against predation (Holzberg, 1978; Konings, 2007; Roberts et al., 2009). In nature, this could asymmetrically favor females, as males with this phenotype are almost never present (Streelman et al., 2003; Roberts et al., 2009). The rarity of OB males can be explained by reduced reproductive fitness, as $\mathrm{OB}$ presumably disrupts recognized male nuptial coloration (Holzberg, 1978; Seehausen et al., 1999b; Kocher, 2004). Tight linkage between OB and ZW could have evolved in response to sexual conflict and might indicate the invasion of this sex system into an ancestral XY (Kocher, 2004; van Doorn and Kirkpatrick, 2007; Roberts et al., 2009; Ser et al., 2010). Intriguingly, because $\mathrm{W}$ shows higher penetrance for color pattern $(\mathrm{OB})$ than for sex (that is, ZW individuals are always OB but can be male when they inherit $\mathrm{Y}$ alleles from other loci (above)), male OB offspring will continue to be produced in mbuna populations segregating multiple sex systems.

Blue color is emergent in these $\mathrm{F}_{2}$; it is absent in the parental individuals but is present in males of $P$. elongatus (the dam's species) and many other LM cichlid species. The blue-barred phenotype is an important male nuptial color conferring mating success (van Oppen et al., 1998; Svensson et al., 2011). We report that blue color is found exclusively in male $\mathrm{F}_{2}$, of both the $\mathrm{BB}$ and $\mathrm{OB}$ type (Figure 3 ). Blue OB may not be well recognized by females in the wild as the blotches disrupt the recognized male pattern (that is, blue-barred; Holzberg, 1978; Seehausen et al., 1999b; Roberts et al., 2009). After accounting for the effect of sex, we identify QTL for blue color on multiple chromosomes, including chromosome 5 (@27.9) near the ZW locus, and chromosome 7 (@44.2) at the putative $\mathrm{X}^{\prime \prime} \mathrm{Y}^{\prime \prime}$. Male coloration and other display traits are known to be in strong linkage with sex chromosomes in several other fish species (Lindholm and Breden, 2002; Basolo, 2006). Sexually antagonistic fitness drives such linkages because trait expression in males is beneficial for reproduction but detrimental to females due to increased predation and/or intraspecific aggression (Bull, 1983; Rice, 1987). Our data thus identify and bolster existing data supporting sex chromosome linkage for colors and patterns with presumed female versus male reproductive function. As the $\mathrm{OB}$ phenotype is putatively beneficial to females, and thus in tight linkage with the ZW locus, it is possible that linkage between sex loci and the blue phenotype is also a result of sexually antagonistic selection.

We interpret these findings in the context of Malawi cichlid evolution, within the confines of one important caveat. Because we map loci contributing to sex and color in an intergeneric cross, the loci and interactions we identify could be the result of this particular hybrid genetic background (Presgraves, 2007) and might not reflect the genetic basis of these phenotypes within natural populations. Generally, the consistency of our data with previous experiments between and within other Malawi rock-dwelling species (Albertson, 2002; Streelman et al., 2003; Roberts et al., 2009; Ser et al., 2010) and 
the peculiar genomic biology of Malawi groups (Loh et al., 2008; Stelkens et al., 2010) tends to argue against this interpretation. Furthermore, because the intercross we studied is made between species that likely exchange genes in the wild (as many rock-dwellers do), our findings are relevant to cichlid evolutionary dynamics.

Multifactorial sex systems are predicted to be unstable and thus transient (Bull and Charnov, 1977; Karlin and Lessard, 1986). Based on the diversity and lability of sex systems in fish lineages and the rapidity and extent of the LM cichlid radiation, the presence of such a complex assortment of sex determiners is perhaps not unexpected (Volff and Schartl, 2001; Nanda et al., 2003; Kondo et al., 2004; Otake et al., 2006; Cnaani et al., 2008; Ross et al., 2009; Ser et al., 2010). We note that Cnaani et al, (2008) have reported a ZW locus for sex on chromosome 3 in the ancestral tilapia (10-20 million years divergent from LM species flock). This $\mathrm{ZW}$ may correspond with the $\mathrm{Z}^{\prime} \mathrm{W}^{\prime}$ described here (3@27.0), suggesting that at least some of these sexdetermining loci are evolutionarily conserved. Theory suggests that certain conditions may favor equilibrium and maintenance of both XY and WZ systems in the same genome (van Doorn and Kirkpatrick, 2010) Additional study is needed in mbuna cichlids, as well as from other evolutionary lineages in Malawi (and elsewhere in East Africa), to determine whether the sex systems identified here (and/or others) segregate widely across this species flock.

\section{DATA ARCHIVING}

SNP marker sequences are available in GenBank on the dbSNP website (accession numbers 51975064-519751979).

\section{CONFLICT OF INTEREST}

The authors declare no conflict of interest.

\section{ACKNOWLEDGEMENTS}

We thank members of the Streelman lab, Reade Roberts, Mark Hay, Joshua Weitz, Michael Goodisman and two anonymous reviewers for comments on previous drafts of this manuscript and the NSF (IOS 0546423) for support.

Albertson RC (2002). Genetic Basis of Adaptive Radiation in East African Cichlid Fishes. $\mathrm{PhD}$ thesis, University of New Hampshire, Durham, $\mathrm{NH}$.

Albertson RC, Kocher TD (2005). Genetic architecture sets limits on transgressive segregation in hybrid cichlid fishes. Evolution 59: 686-690.

Albertson RC, Streelman JT, Kocher TD, Yelick PC (2005). Integration and evolution of the cichlid mandible: the molecular basis of alternative feeding strategies. Proc Natl Acad Sci USA 102: 16287-16292.

Amorim MCP, Knight ME, Stratoudakis Y, Turner GF (2004). Differences in sounds made by courting males of three closely related Lake Malawi cichlid species. J Fish Biol 65: 1358-1371.

Amorim MCP, Simoes JM, Fonseca PJ, Turner GF (2008). Species differences in courtship acoustic signals among five Lake Malawi cichlid species (Pseudotropheus spp.). J Fish Biol 72: 1355-1368.

Baroiller JF, D'Cotta H, Saillant E (2009). Environmental effects on fish sex determination and differentiation. Sex Dev 3: 118-135.

Basolo AL (2006). Genetic linkage and color polymorphism in the southern platyfish (Xiphophorus maculatus): a model system for studies of color pattern evolution. Zebrafish 3: 65-83.

Broman KW, Sen S (2009). A Guide to QTL Mapping with R/qtl. Springer: New York, N.Y.

Bull JJ (1983). Evolution of Sex Determining Mechanisms. Benjamin Cummings: Menlo Park, CA.

Bull JJ, Charnov EL (1977). Changes in the heterogametic mechanism of sex determination. Heredity 39: 1-14.

Carleton K (2009). Cichlid fish visual systems: mechanisms of spectral tuning. Int Zool 4: $75-86$.

Carroll SB (2000). Endless forms: the evolution of gene regulation and morphological diversity. Cell 101: 577-580.

Chapman T (2006). Evolutionary conflicts of interest between males and females. Curr Biol 16: R744-R754.

Cnaani A, Lee BY, Zilberman N, Ozouf-Costaz C, Hulata G, Ron H et al. (2008). Genetics of sex determination in tilapiine species. Sex Dev 2: 43-54.
Cole TB, Stacey NE (2006). Olfactory responses to steroids in and African mouth-brooding cichlid, Haplochromis burtoni. J Fish Biol 68: 661-680.

Danley PD, Husemann M, Chetta J (2011). Acoustic diversity in Lake Malawi's rockdwelling cichlids. Env Biol Fish 75: 23-30.

Ezaz T, Stiglec R, Veyrunes F, Graves JAM (2006). Relationships between vertebrate ZW and XY sex chromosome systems. Curr Biol 16: R736-R743.

Fan JB, Oliphant A, Shen R, Kermani BG, Garcia F, Gunderson KL et al. (2003). Highly parallel SNP genotyping. Cold Spring Har Symp Quant Biol 68: 69-78.

Genner MJ, Turner GF (2005). The mbuna cichlids of Lake Malawi: a model for rapid speciation and adaptive radiation. Fish Fisheries 6: 1-34.

Gerlai R (2007). Mate choice and hybridization in Lake Malawi cichlids, Sciaenochromis fryeri and Cynotilapia afra. Ethology 113: 673-685.

Holzberg S (1978). A field and laboratory study of the behaviour and ecology of Pseudotropheus zebra (Boulenger), an endemic cichlid of Lake Malawi (Pisces: Cichlidae). Zeit Zool Syst Evol 16: 171-187.

Jordan R, Kellogg K, Juanes F, Stauffer Jr J (2003). Evaluation of female mate choice cues in a group of Lake Malawi Mbuna (Cichlidae). Copeia 2003: 181-186.

Karlin S, Lessard S (1986). Sex Ratio Evolution. Princeton University Press;: Princeton, NJ.

Kitano J, Ross JA, Mori S, Kume M, Jones FC, Chan YF et al. (2009). A role for a neo-sex chromosome in stickleback speciation. Nature 461: 1079-1083.

Knight ME, Turner GF (2004). Laboratory mating trials indicate incipient speciation by sexual selection among populations of the cichlid fish Pseudotropheus zebra from Lake Malawi. Proc R Soc Lond B 271: 675-680.

Kocher TD (2004). Adaptive evolution and explosive speciation: the cichlid fish model. Nat Rev Gen 5: 288-298.

Kondo M, Nanda I, Hornung U, Schmid M, Schartl M (2004). Evolutionary origin of the medaka Y chromosome. Curr Biol 14: 1664-1669.

Konings A (2007). Malawi Cichlids in their Natural Habitat, 4th edn Cichlid Press: El Paso, TX.

Lande R, Seehausen O, van Alphen JJ (2001). Mechanisms of rapid sympatric speciation by sex reversal and sexual selection in cichlid fish. Genetica 112-113: 435-443.

Lee B-Y, Lee W-J, Streelman JT, Carleton KL, Howe AE, Hulata G et al. (2005). A secondgeneration genetic linkage map of tilapia (Oreochromis spp.). Genetics 170: 237-244.

Lindholm A, Breden F (2002). Sex chromosomes and sexual selection in poeciliid fishes. Am Nat 160: S214-S224.

Loh YE, Katz LS, Mims MC, Kocher TD, Yi SV, Streelman JT (2008). Comparative analysis reveals signatures of differentiation amid genomic polymorphism in Lake Malawi cichlids. Gen Bio/ 9: R113.

Maan ME, Seehausen O, Söderberg L, Johnson L, Ripmeester EAP, Mrosso HDJ et al. (2004). Intraspecific sexual selection on a speciation trait, male coloration, in the Lake Victoris cichlid Pundamilia nyererei. Proc $R$ Soc Lond B 271: 2445-2452.

Mank JE, Promislow DEL, Avise JC (2006). Evolution of alternative sex-determining mechanisms in teleost fishes. Biol J Linn Soc 87: 83-93.

Matsuda M, Nagahama Y, Shinomiya A, Sato T, Matsuda C et al. (2002). DMY is a Y-specific DM-domain gene required for male development in the medaka fish. Nature 417: 559-563.

Meiklejohn CD, Tao Y (2010). Genetic conflict and sex chromosome evolution. Trends Ecol Evol 25: 215-223.

Mims MC, Hulsey CD, Fitzpatrick BM, Streelman JT (2010). Geography disentangles introgression from ancestral polymorphism in Lake Malawi cichlids. Mol Ecol 19 : 940-951.

Nanda I, Hornung U, Kondo M, Schmid M, Schartl M (2003). Common spontaneous sexreversed XX males of the medaka Oryzias latipes. Genetics 163: 245-251.

Nanda I, Kondo M, Hornung U, Asakawa S, Winkler C, Shimizu A et al. (2002). A duplicated copy of DMRT1 in the sex-determining region of the Y-chromosome of the medaka, Oryzias latipes. Proc Natl Acad Sci USA 99: 11778-11783.

Oliphant A, Barker DL, Stuelpnagel JR, Chee MS (2002). BeadArray technology: enabling an accurate, cost-effective approach to high-throughput genotyping. Biotechniques 32 S56.

Otake H, Shinomiya A, Matsuda M, Hamaguchi S, Sakaizumi M (2006). Wild-derived XY sex-reversal mutants in the medaka, Oryzias latipes. Genetics 173: 2083-2090.

Parnell NF, Hulsey CD, Streelman JT (2008). Hybridization produces novelty when mapping of form to function is many to one. BMC Evol Biol 8: 122-132.

Parnell NF, Hulsey CD, Streelman JT (2012). The genetic basis of a complex functional system. Evolution (doi:10.1111/j.1558-5646.2012.01688.x).

Parnell NF, Streelman JT (2011). The macroecology of rapid evolutionary radiation. Proc $R$ Soc Lond B 278: 2486-2494.

Plenderleith M, van Oosterhout C, Robinson RL, Turner GF (2005). Female preference for conspecific males based on olfactory cues in a Lake Malawi cichlid. Biol Lett 1 : 411-414.

Presgraves DC (2007). Speciation genetics: epistasis, conflict and the origin of species. Curr Biol 17: R125-R127.

Qvarnstrom A, Bailey RI (2009). Speciation through evolution of sex-linked genes. Heredity 102: 4-15.

Ribbink AJ, Marsh AC, Ribbink CC, Sharp BJ (1983). A preliminary survey of the cichlid fishes of rocky habitats in Lake Malawi. S Afr J Zool 18: 149-310.

Rice WR (1987). The accumulation of sexually antagonistic genes as a selective agent promoting the evolution of reduced recombination between primitive sex chromosomes. Evolution 41: 911-914.

Rice WR (1992). Sexually antagonistic genes - experimental evidence. Science 256: 1436-1439.

Rice WR (1998). Male fitness increases when females are eliminated from gene pool: implications for the Y chromosome. Proc Natl Acad Sci USA 95: 6217-6221. 
Roberts RB, Ser JR, Kocher TD (2009). Sexual conflict resolved by invasion of a novel sex determiner in Lake Malawi cichlid fishes. Science 326: 998-1001.

Ross JA, Urton JR, Boland J, Shapiro MD, Peichel CL (2009). Turnover of sex chromosomes in the stickleback fishes (Gasterosteidae). PLoS Genet 5: e1000391.

Ruber L, Meyer A, Sturmbauer C, Verheyen E (2001). Population structure in two sympatric species of the Lake Tanganyika cichlid tribe Eretmodini: evidence for introgression. Mol Ecol 10: 1207-1225.

Salzburger W, Baric S, Sturmauer C (2002). Speciation via introgressive hybridization in East African cichlids? Mol Ecol 11: 619-625.

Seehausen 0 (2004). Hybridization and adaptive radiation. Trends Ecol Evol 19 198-207.

Seehausen $O$ (2006). African cichlid fish: a model system in adaptive radiation research. Proc Roy Soc B 273: 1987-1998.

Seehausen O, Mayhew PJ, van Alphen JJM (1999b). Evolution of colour patterns in East African cichlid fish. J Evol Biol 12: 514-534.

Seehausen O, Terai Y, Magalhaes IS, Carleton KL, Mrosso HDJ, Miyagi R et al. (2008). Speciation through sensory drive in cichlid fish. Nature 455: 620-627.

Seehausen O, van Alphen JJM, Lande R (1999a). Color polymorphism and sex ratio distortion in a cichlid fish as an incipient stage in sympatric speciation by sexual selection. Ecol Lett 2: 367-378.

Seehausen O, van Alphen JJM, Witte F (1997). Cichlid fish diversity threatened by eutrophication that curbs sexual selection. Science 277: 1808-1811.

Ser JR, Roberts RB, Kocher TD (2010). Multiple interacting loci control sex determination in Lake Malawi cichlid fish. Evolution 64: 486-501.

Simoes JM, Fonseca PJ, Turner GF, Amorim MCP (2008). African cichlid Pseudotropheus spp. males moan to females during foreplay. J Fish Biol 72: 2689-2694.

Smith AR, van Staaden MJ (2009). The association of visual and acoustic courtship behaviors in African cichlid fishes. Mar Fresh Behav Phys 42: 211-216.

Smith PF, Konings A, Kornfield I (2003). Hybrid origin of a cichlid population in Lake Malawi: implications for genetic variation and species diversity. $\mathrm{Mol} E \mathrm{Eol} 12$ : 2497-2504.
Smith PF, Kornfield I (2002). Phylogeography of Lake Malawi cichlids of the genus Pseudotropheus: significance of allopatric colour variation. Proc R Soc Lond B 269 2495-2502.

Stelkens RB, Young KA, Seehausen O (2010). The accumulation of reproductive incompatibilities in African cichlid fish. Evolution 64: 617-633.

Streelman JT, Albertson RC, Kocher TD (2003). Genome mapping of the orange blotch colour pattern in cichlid fishes. Mol Ecol 12: 2465-2471.

Streelman JT, Gmyrek SL, Kidd MR, Kidd C, Robinson RL, Hert E et al. (2004). Hybridization and contemporary evolution in an introduced cichlid fish from Lake Malawi National Park. Mol Ecol 13: 2471-2479.

Streelman JT, Peichel CL, Parichy DM (2007). Developmental genetics of adaptation in fishes: the case for novelty. Ann Rev Ecol Evol Syst 38: 655-681.

Svensson O, Egger B, Gricar B, Woodhouse K, van Oosterhout C, Salzburger W et al. (2011). Segregation of species-specific male attractiveness in $F_{2}$ hybrid Lake Malawi cichlid fish. Int J Evol Biol 2011: 1-7.

van Doorn GS, Kirkpatrick M (2007). Turnover of sex chromosomes induced by sexual conflict. Nature 449: 909-912.

van Doorn GS, Kirkpatrick M (2010). Transitions between male and female heterogamety caused by sex-antagonistic selection. Genetics 186: 629-645.

van Ooijen JW, Voorrips RE (2001). Joinmap 3.0, Software for the Calculation of Genetic Linkage Maps. Plant Research International: Wageningen, Netherlands.

van Oppen MJH, Turner GF, Rico C, Robinson RL, Deutsch JC, Genner MJ et al. (1998). Assortative mating among rock-dwelling cichlid fishes supports high estimates of species richness from Lake Malawi. Mol Ecol 7: 991-1001.

Volff J-N, Schartl M (2001). Variability of genetic sex determination in poeciliid fishes. Genetica 111: 101-110.

Wilkins AS (1995). Moving up the hierarchy: a hypothesis on the evolution of a genetic sex determination pathway. Bioessays 17: 71-77.

Won Y-J, Wang Y, Sivasundar A, Hey J (2005). On the origin of Lake Malawi cichlid species. Proc Natl Acad Sci USA 102: 6581-6586.

Yoshida K, Terai Y, Mizoiri S, Aibara M, Nishihara H, Watanabe M et al. (2011). B chromosomes have a functional effect on female sex determination in Lake Victoria cichlid fishes. Plos Genet 7: e1002203.

Supplementary Information accompanies the paper on Heredity website (http://www.nature.com/hdy) 